Int. J. Dev. Biol. 51: 37-44 (2007)

doi: $10.1387 /$ ijdb.052108sl

Original Article

\title{
Blood vessel/epicardial substance (bves) expression, essential for embryonic development, is down regulated by Grk/EFGR signalling
}

\author{
SHENGYIN LIN ${ }^{1}$, DEBIAO ZHAO ${ }^{2}$ and MARY BOWNES* \\ Institute of Cell Biology, School of Biological Sciences, University of Edinburgh, U.K.
}

\begin{abstract}
The Pop1/Bves (blood vessel/epicardial substance) gene is a member of the popeye gene family recently identified in various species. It encodes a potential transmembrane glycoprotein and is a cell adhesion molecule present in skeletal and cardiac muscle and epithelia. We isolated the Drosophila homologue of Bves (DmBves) and found, using in situ hybridisation to RNA in ovaries, that bves is expressed in all follicular epithelial cells surrounding the oocyte at stage 10, except those in very posterior and anterior-dorsal regions adjacent to the oocyte. We show that the repression of bves expression in anterior-dorsal follicle cells is regulated by the Grk/ EGFR signalling pathway. Bves is also expressed in nurse cells during oogenesis and its transcripts are then translocated into the oocyte. Expression of bves antisense RNA during oogenesis causes reduced viability in the resulting embryos. There is a failure in the migration of pole cells from the posterior towards the antero-dorsal side of the embryo, probably resulting from abnormal germband extension and we suggest that bves is essential for normal embryonic development.
\end{abstract}

KEY WORDS: bves, Grk signalling, Drosophila, oogenesis, embryonic development

\section{Introduction}

During early Drosophila oogenesis, one germ line stem cell generates 16 cystocytes by dividing four times. Only one of these cystocytes is determined to be an oocyte and the rest become nurse cells (Telfer, 1975). Many of the RNAs and proteins important for early embryogenesis are synthesized in the nurse cells and then transported into the oocyte at various stages in its development (Spradling, 1993). These include bicoid RNA and Dorsal protein essential for the establishment of the embryonic axis; and osk and nanos RNA needed for determination of germ cells (Riechmann and Ephrussi, 2001). The development of a polarized egg requires intercellular communication between the oocyte and somatic follicle cells surrounding it. The transcripts of gurken (grk), a Transforming Growth Factor (TGF)- $\alpha$ homologue are translocated to the posterior of the oocyte which transmits the Grk signal to the adjacent posterior follicle cells (Gonzalez-Reyes, et al., 1995). Epidermal Growth Factor Receptor (EGFR) encoded by the torpedo (top) gene is then activated via the Ras signalling pathway and the follicle cells located at the posterior of the oocyte adopt a posterior follicle cell fate. The posterior follicle cells then signal back to the oocyte, mediated by the Protein kinase $A(P K A)$, merlin and Laminin $A$ genes and this leads to the reorganization of microtubules and results in a relocalization of grk RNAs to the anterior-dorsal region of the oocyte (Deng and Ruohola-Baker, 2000). This is crucial for the establishment of the anterior/posterior axis of the oocyte and embryo. The oocyte then sends the Grk signal to the adjacent follicle cells which adopt an anterior-dorsal fate, again via the EGFR and Ras pathways. These anterior-dorsal follicle cells will later mainly secrete and model the dorsal appendages, thus their signal is key to establishing the dorsal/ventral axis of the egg. The remaining ventral follicle cells later set up a ventral signal which is required to activate Dorsal protein to generate the dorsal-ventral axis of the future embryo.

Abbreviations used in this paper: bves, blood vessel/epicardial substance gene; EGFR, epidermal growth factor repceptor; Grk, gurken; PKA, protein kinase A; pop, popeye gene.

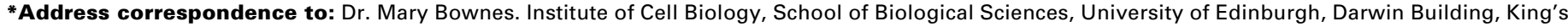
Buildings, Mayfield Road, Edinburgh, EH9 3JR, UK. Fax: +44-131-650-5371. e-mail: mary.bownes@ed.ac.uk
}

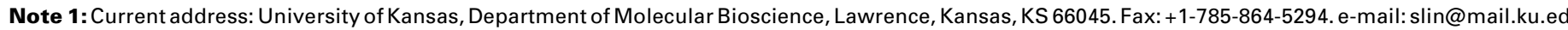
Note 2: Current address: Roslin Institute, Roslin, Midlothian, Edinburgh, EH25 9PS, UK. Fax: +44-131-440-0434. e-mail: DB.Zhao@bbsrc.ac.uk 
During embryonic gastrulation, a series of morphogenetic events occur, including germ band extension. It is initiated by proctodeal invagination and followed by movement of the posterior midgut primordium from the posterior along the anteriordorsal side. Pole cells move with the posterior midgut primordium and the pole cells later leave the midgut by traversing its epithelium to find the somatic gonadal precursor cells (Warrior, 1994, Jaglarz and Howard, 1994). Later germ band retraction begins and as a consequence, the yolk sac is pushed to the dorsal side of embryo, being only covered by the amnioserosa. Morphological analysis of germ band extension has helped to identify many genes involved in the process, including genes affecting dorsal-ventral polarity, but few genes have been found that affect germ band retraction (Costa, et al., 1993).

Three popeye genes encoding putative transmembrane proteins have been recently identified, Pop1, Pop2 and Pop3 in human and mouse and Pop1 and Pop3 in chicken (Andree, et al., 2000; Reese and Bader, 1999). Pop1 generates four different transcripts (Pop1A to Pop1D). The Bves (blood vessel/ epicardial substance) gene was the first member of the popeye gene family to be found and is identical to Pop $1 A$. The strong conservation of pop genes in various species suggests that they may have an important conserved function. However, homozygous mice lacking pop1 were fertile and had a normal life span without any apparent phenotype except defects in skeletal muscle regeneration (Andree, et al., 2002).

To better understand the possible role of popeye genes, the expression of Pop 1 has been investigated in different animals. High levels of Pop 1 transcripts were detected in both cardiac and skeletal muscle of human, mouse, chicken and frog by Northern blots and by in situ hybridisation to RNA (Andree, et al., 2000, Hitz et al., 2002 and Reese and Bader, 1999). The expression of the Pop1 gene in the muscles was further confirmed by Pop1-lacZ expression during mouse development (Andree, et al., 2002). However, staining with antibody against Bves (Pop1) has shown wider expression in variety of tissues (Andree, et al., 2000, Reese, et al., 1999, Osler and Bader, 2004, Vasavada, 2004 and DiAngelo, etal., 2001). Bves protein was originally observed in the epicardial and smooth muscle of coronary arteries in the chicken (and hence called blood vessel/ epicardial substance) (Reese, et al., 1999) and has subsequently been observed in cardiac and skeletal muscle, as well as additional epithelial cells in both mouse and chicken (Andree, et al., 2000, Vasavada, 2004 and Wada et al., 2001). More recently chicken Bves protein was found to be expressed in epithelia of all three germ layers early in development, as well as in epithelial tissues during organogenesis, specifically the developing epidermis, the gut endoderm and the epicardium of the heart (Osler and Bader, 2004). Using human corneal epithelial cells, Bves staining was observed at the epithelial surface (Ripley et al., 2004).

Study of pop1/Bves at the molecular and cellular level sheds light on how it is involved in cell adhesion and movement. Bves is a membrane protein with three predicted transmembrane helices and two asparagine-linked glycosylation sites within the amino terminus (Andree, et al., 2000 and Wada et al., 2001). Membrane topology of the cell surface shows Bves is composed of a glycosylated extracellular amino terminus and a cytoplasmic carboxyl terminus (Knight et al., 2003). Bves accu- mulates in a perinuclear region in disassociated epicardial cells until cells make contact, at which point Bves is trafficked to the cell membrane (Wada et al., 2001). Bves accumulated at points of cell/cell contact, such as filopodia or cell borders and can confer adhesive behaviour to L-cells after transfection. Furthermore, Bves antibodies inhibited epithelial migration of vasculogenic cells from the proepicardium (Wada et al., 2001). Recently, monoclonal antibodies against chicken Pop1/Bves have been used to demonstrate the presence of the protein in cardiomycytes and confirm its membrane location (Vasavada, et al., 2004). All these data suggest that Pop1/Bves is involved in cell adhesion and cell movement in early embryogenesis.

In this paper, we describe the identification of a Pop1/Bves homologue in Drosophila melanogaster (bves). bves is expressed all epithelial follicle cells surrounding the oocyte except those in anterior-dorsal and very posterior regions. Its repression in anterior-dorsal epithelial cells is due to Grk/EGFR signalling. bves is also expressed in the nurse cells and its transcripts are transported into the oocyte. Expression of bves antisense RNA during oogenesis caused the failure of migration of pole cells from the posterior towards the anterior-dorsal side of embryo as well as embryonic death. This study provides evidence that Bves is required for cell migration and is essential for embryonic development in Drosophila.

\section{Results}

Isolation and molecular characterisation of the bves gene

The bves gene was identified as part of a GAL4/UAS enhancer trap scheme to find genes involved in the generation of dorsal ventral polarity during Drosophila oogenesis. When line C648, a GAL4 line, is crossed with a UAS-lacZ line, staining is detected in a large patch of follicle cells at stage 10 of oogenesis (Fig. 2A). Cloning of the target gene was achieved by plasmid rescue (Bellen et al., 1989). The cDNA isolated was named bves (Drosophila melanogaster bves) as its vertebrate homologue is called blood vessel/epicardial substance (Bves). Two EST clones (GM07524 and LD22978) were found that were similar to bves cDNA. GM07524 and LD22978 have inserts of $2.6 \mathrm{~kb}$ and $5.5 \mathrm{~kb}$ respectively. These two EST clones were sequenced and revealed that LD22978 has an insert containing two genes. The 5 ' fragment of $2.6 \mathrm{~kb}$ has a similar sequence to GM07528. The rest of the sequence of LD22978 is from another putative gene named CG5208, which is located on chromosome 3. We assumed EST GM07524 to be a complete cDNA and we submitted the bves DNA sequence we obtained to genebank (Accession No: AF247183, Oct. $\left.3^{\text {rd }}, 2000\right)$. The sequence is not therefore presented again in this paper.

To learn more about the function of the bves gene, we need to know the developmental expression profile of its transcript and this was determined by northern blotting. The results revealed that bves transcripts are present at various developmental stages (Fig. 1C). The transcript of bves was found to be $4 \mathrm{~kb}$ long, which was not consistent with the bves cDNA (2.6kb) and the length of the transcript predicted from EST GM07524. This problem was resolved by the latest Drosophila genome annotation, which predicts that the bves cDNA is $3.9 \mathrm{~kb}$ long from EST RE59473. This is consistent with the transcript slice we observed by Northern blots. 
Comparing the Drosophilagenomic sequence with the bves cDNA sequence established that the bves gene is $24 \mathrm{~kb}$ with 7 exons (Fig 1). It is located on chromosome $X$ at position $19 \mathrm{E} 7$. There is a large intron which is approximately $14 \mathrm{~kb}$ between exon 2 and exon 3 . The P-element insertion in the original line (C648) was mapped by sequencing the rescued genomic DNA generated by plasmid rescue and we showed that it is inserted

A

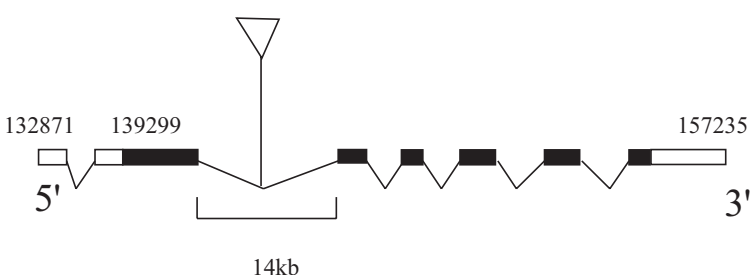

B

human mouse chicken Dmbves mosquito in the middle of the large intron at basepair 146195 (Fig. 1A). The bves gene encodes a single ORF and is translated from $680 \mathrm{bp}$ to $1924 \mathrm{bp}$, producing a peptide of 414 amino acids. The amino acid sequence of bves was used to search the NCBI database. Three similar genes were found in mouse and humans called popeye, Pop1 to Pop3 and two in chicken, Bves and Pop3. In the Drosophila genome, there is one homologue of the popeye gene. We named the Drosophila homologue bves. There is approximately $28 \%$ identity and $50 \%$ similarity at the protein sequence level between bves and its homologues. Later, homologues of bveswere found in the mosquito (Ensembl, European Bioinformatics Institute 2004). The amino acid sequences show high identity (59\%) and similarity (71\%) between these two insects. Like other Pop1/Bves homologues, the predicted bves sequence contains protein that has three transmembrane domains and one glycosylation site at the amino terminus. It could therefore function an adhesion molecule in a similar wav to its homoloaues. The predicted protein seauences
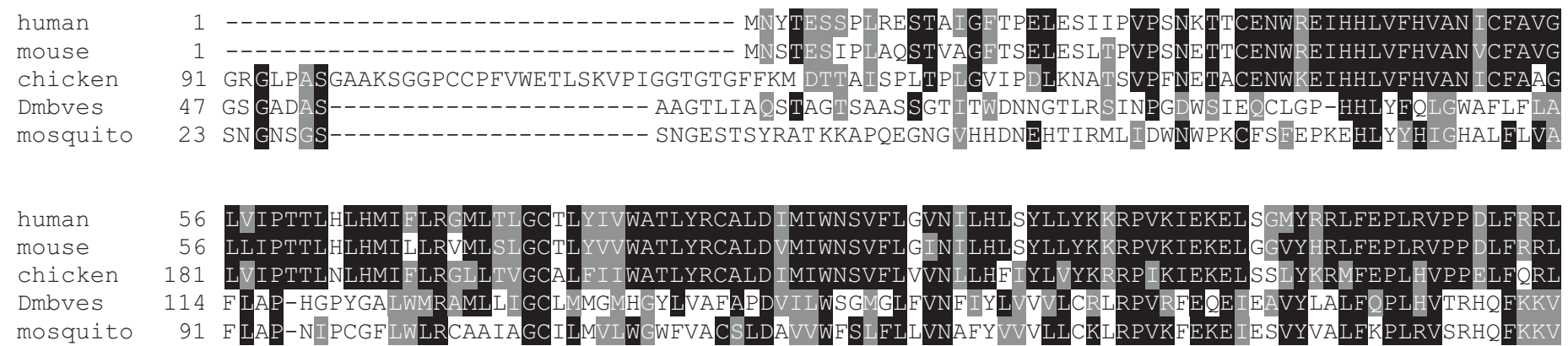

human mouse chicken Dmbves mosquito
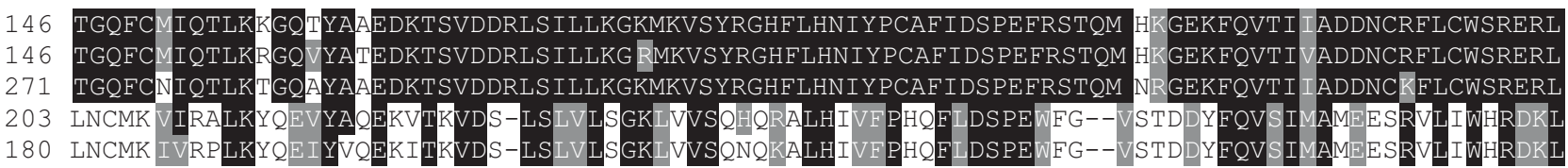

human mouse chicken Dmbves mosquito

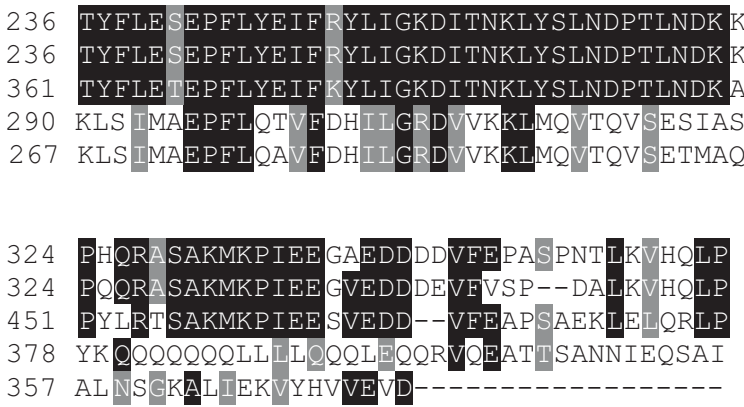

human mouse chicken Dmbves mosquito

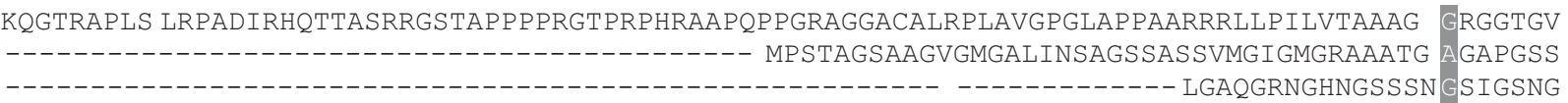

C

$$
\begin{array}{lllllll}
1 & 2 & 3 & 4 & 5 & 6 & 7
\end{array}
$$

$4 \mathrm{~kb}$

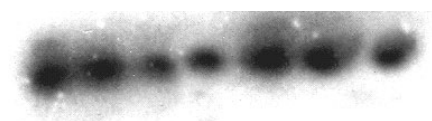

The bves gene. (A) The intron/exon structure of the bves gene. The boxes are exons. Black represents the coding regions. The triangle indicates that the P-element is inserted between exon 2 and exon 3. The numbers in the figure correspond to the base pairs in the genome. (B) Alignment of protein sequences encoded by the bves gene and the mouse, chicken, mosquito and human (Bves) homologues. Amino acids shaded in black are identical and those shaded in grey are similar in these five sequences. (C) Total RNAs were extracted from different developmental stages. Lane 1, ovaries; Lane 2, 0-4 h embryos; Lane 3, 6-14 h embryos; Lane 4, first instar larvae; Lane 5, second instar larvae; Lane 6, third instar larvae; Lane 7, pupae. bves DNA was used as the probe. $20 \mu \mathrm{g}$ total RNA of each sample was loaded on the gel. 

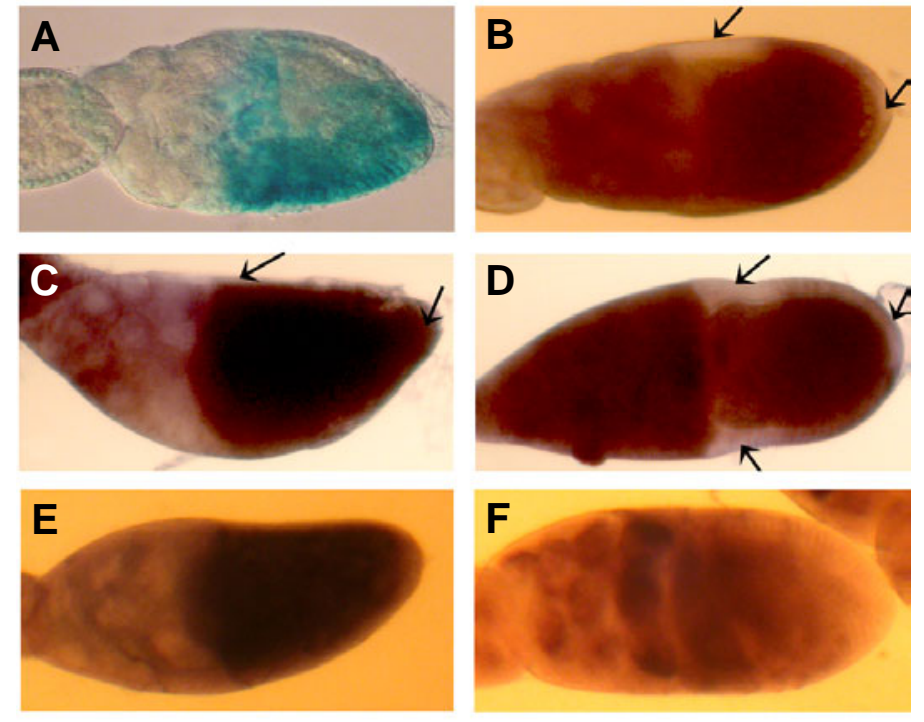

I II III

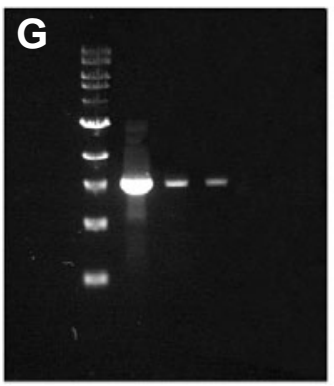

Fig. 2. $\beta$-galactosidase staining and RNA in situ hybridisaton to RNA in the ovary. (A) $\beta$-galactosidase staining in the ovary. A large patch of follicle cells was stained. (B) In situ hybridization to the wild type ovary, showing expression in nurse cells and all follicle cells except in the anterior-dorsal and posterior regions (arrows). (C) Expression of bves in gurken mutant ovaries. Arrows indicate that bves is expressed in all follicle cells. (D) Expression of bves in fs(1) K10 mutant ovaries. Arrows indicate that bves is not expressed in a stripe along the anterior region of the oocyte. (E) Expression of bves antisense RNA in PCas-hs-bves-AS (2) ovaries. (F) Expression of bves antisense RNA in PCas-hs-bves- AS (40) ovaries. Anterior is to the left and dorsal is at the top. (G) RT-PCR to detect bves RNA in ovaries. I, RNA from wild type ovaries; II, RNA from heatshocked PCas-hs-bves- AS (40) ovaries; III, RNA from PCas-hs-bves-AS (2) ovaries.

of Pop1/Bves were aligned between bves, the chicken, mouse, mosquito and human homologues and this is shown in Fig. 1B.

\section{The repression of DmBves expression in some follicle cells is caused by Grk/EGFR signalling during oogenesis}

To learn more about the bvesgene and its role in oogenesis and embryogenesis, in situhybridisation to bves transcripts using bves cDNA as a probe was carried out using wild type ovaries. A similar, but not identical, expression pattern in the follicle cells was observed when comparing the expression pattern of a reporter gene driven by the GAL4 insert in line C648. Differences are probably because the insert containing GAL4 only responds to some of the regulatory elements that affect the expression of the native gene. Expression was observed in the nurse cells from stage 5 onwards. Transient expression was seen in all follicle cells surrounding the oocyte at stage 10 of oogenesis, except those located at the anterior-dorsal and posterior region (Fig. 2B). There was no detectable expression in follicle cells at any stages before or after stage 10 of oogenesis, this expression is therefore very transient and the mRNA seems to be short-lived. During oogenesis grk signals to the follicle cells in the posterior region at stage 6 and signals again to the follicle cells in the anterior-dorsal region at stage 10 to determine their fates. It is therefore possible that Grk signals to an unknown gene and that the products of this gene may repress bves expression in these groups of follicle cells. Although Grk is located in the anterior-dorsal region at stage 10 , it is possible that the products of the unknown gene remain in the posterior follicle cells for some time and repress bves expression.

To investigate if the bves expression pattern is dependent upon the Grk/EGFR pathway, in situ hybridisation to the ovaries of a number of mutants that affect the Grk/EGFR signalling pathway was carried out. In the ovaries of the grk homozygous female mutant, follicle cells do not receive the Grk signal either in the posterior or anterior-dorsal region and as a result, the expression of those genes downstream of Grk/EFFR signalling pathway is affected. Using bves as a probe for RNA in situhybridization in the grkmutant ovaries, we found that bveswas expressed in all follicle cells surrounding the oocyte (Fig. $2 \mathrm{C}$ ), indicating that bves expression is negatively regulated by Grk/EGFR signalling. In $f_{s}(1)$ K10 mutant ovaries, grk transcripts diffuse from their normal anteriordorsal location towards anterior-ventral positions within the oocyte, therefore all anterior follicle cells receive the Grk signal. In situ hybridisation to $f s(1) K 10$ mutant ovaries with bves as a probe revealed that bves was not expressed at all in any anterior follicle cells (Fig. 2D). This data taken together suggests that the Grk/ EGFR signalling pathway is used to establish the specific expression pattern of the bvesgene in follicle cells surrounding the oocyte during oogenesis. In situ hybridisation was also carried out using wild type embryos, bvestranscripts are detected in the embryo but there are no regions or tissues with higher expression (data not shown). Since developmental northerns show that bves RNAs is present levels in 0-4 hour and 6-14 hour embryos (Fig. 1C) It seems likely that bvesmRNA distributed uniformly in the embryo. It will be crucial in future, once antibodies to DrosophilaBVES are available to investigate the protein distribution, which may well move tissue variations or locations in specific regions within cells.

\section{Functional studies}

There are no bves mutant lines available. To investigate the biological function of bves, P-element mobilisation was carried out on fly line $\mathrm{C} 648$ in the hope of generating mutants of the bvesgene. 316 balanced lines were established. Among them 156 were red eyed lines in which the P-element was relocated and 160 were white eyed lines in which the P-element had at least partially excised. Only four of the red-eyed lines were been found to be homozygous lethal. There are no homozygous lethal lines among the white eyed lines. No other visible mutants were found in either the red-eyed line or the white eyed lines, such as female steriles, or abnormal phenotypes of the adult flies. The reason why there are no mutant flies amongst the white eyed lines may be that the Pelement is inserted in the middle of a large intron. The P-element may have removed some flanking genomic DNA when excising imprecisely, but not enough genomic DNA to affect gene expression. 4 red-eyed fly lines which are homozygous lethal were obtained. Southern hybridisation was used to check if the Pelement is really inserted in the genome of these mutant flies, using pbluescript DNA which is contained within the pGawB insect as probe. It was found that the P-element is present in all three lines. To determine the position of the $\mathrm{P}$ insertion, plasmid rescue for these three lines with P-element insertions was carried out and the 
TABLE 1

\section{SURVIVAL OF EMBRYOS LAID BY HEATSHOCKED FEMALES TO DRIVE ANTISENSE EXPRESSION}

\begin{tabular}{llcccc}
$\begin{array}{l}\text { Time after } \\
\text { heatshock }\end{array}$ & Fly lines & $\begin{array}{c}\text { Total } \\
\text { embryos }\end{array}$ & $\begin{array}{c}\text { Dead } \\
\text { embryos }\end{array}$ & $\begin{array}{c}\text { Larvae } \\
\text { hatched }\end{array}$ & $\begin{array}{c}\text { Survival } \\
\text { rate }\end{array}$ \\
\hline First day & OrR - control & 361 & 38 & 323 & $89 \%$ \\
& PCas-hs-bves-AS (2) & 309 & 160 & 149 & $48 \%$ \\
& PCas-hs-bves-AS (40) & 135 & 72 & 63 & $47 \%$ \\
Second day & OrR - control & 150 & 15 & 135 & $90 \%$ \\
& PCas-hs-bves-AS (2) & 164 & 61 & 103 & $63 \%$ \\
\multirow{5}{*}{ Third day } & PCas-hs-bves-AS (40) & 53 & 22 & 31 & $59 \%$ \\
& OrR - control & 69 & 3 & 66 & $96 \%$ \\
& PCas-hs-bves-AS (2) & 123 & 49 & 74 & $60 \%$ \\
& PCas-hs-bves-AS (40) & 76 & 47 & 29 & $38 \%$ \\
\hline
\end{tabular}

The table shows survival of embryos laid on each day following induction of antisense BVES in the ovaries of the mother. The eggs laid first will have had antisense bves RNA present for a shorter period of oocyte development than eggs laid later.

rescued DNA fragments sequenced. The results revealed that the P-element had hopped to positions unfortunately upstream or downstream of Bves gene. Thus there was no homozygous lethal mutant resulting from $P$ insertion in the bves gene, so either the gene is non-essential or redundant or we failed to damage any of the coding regions in any of the excision lines or P-hop lines.

Since no mutants were obtained we generated transgenic fly lines in which bves RNA antisense expression is induced by heatshock to try to establish potential functions of the gene by the antisense RNA knocking down bves transcript levels. To analyse the expression of bvesantisense RNA induced by heatshock in the different transgenic lines the ovaries of the heatshocked flies were collected one hour after the last heatshock. In situhybridisation to RNA in these ovaries was carried out using the bves sense RNA as a probe. Strong expression of bves antisense RNA were observed in two fly lines. The expression level and pattern of bves antisense RNA strand depends upon the chromosomal position of the insert (Karpen, G. H. 1994, Simmons, M.J. et al., 1996). In PCas-hs- bves -AS line 40, bves antisense RNA was expressed strongly in the nurse cells and weakly in follicle cells (Fig. 2F). In the PCas-hs-bves-AS line 2, bves antisense RNA was expressed the other way around, strongly in the follicle cells and weakly in nurse cells (Fig. 2E). The different expression patterns of these two lines is probably related to the position of the P-element insert in the genome since expression patterns will be influenced by nearby enhancers. To see if the heatshocked induced antisense RNA really reduced the endogenous bvesRNA levels, in situhybridisation to the heatshocked ovaries of these two fly lines by using bves antisense RNA as a probe. There is no RNA detected by in situ (data not shown). RT-PCR is more sensitive and was also the reference used to detect bves RNA in the heatshocked ovaries of those two lines. We found that bves RNA still existed but at a much lower level in comparison with that observed in the wild type ovaries (Fig. 2G). Thus line 40 reduced nurse cell expression and line 2 the follicle cell expression more. Levels in all cells of the ovary are below the levels detectable by in situ hybridisation, but some transcripts remain, thus this approach is not leading to a null level of bves.

To investigate the function of bves in oogenesis, the PCas-hsbves-AS line 40 and PCas-hs-bves-AS line 2 were heatshocked and the ovaries were observed. There were no defects detectable in the ovaries thus the eggs laid by heatshocked flies were investigated. They all had normal egg-shells and dorsal appendages. These results were not expected as one might have predicted there would be defects in oogenesis or the morphology of the egg/embryo with the expression patterns observed. It is possible that insufficient antisense RNA was produced in time to affect the phenotype, however, the RT-PCR suggests a significant reduction in the RNA levels. We found that embryos laid by heatshocked wild type females had a much higher survival rate than those laid by bves antisense RNA lines (Table 1). This suggests embryos were not killed by heatshock of the mothers, but by expression bves antisense RNA, leading to reduced levels of native bves RNA during oogenesis. Embryos laid by heatshocked flies (hs embryos) develop normally from stage 1 to 6 , (stages of Campos-Ortega and Hartenstein, 1985; Roberts, 1998). In the wild-type embryos at stage 7 , the cephalic furrow has deepened and the posterior midgut has carried the pole cells to the dorsal side of the embryo (Fig. 3B). But posterior midgut invagination did not occur properly in almost $10 \%$ of the embryos laid by females expressing bves antisense RNA and sometimes pole cells were left behind at the posterior end (Fig. 3D). At stage 8, in wild-type embryos the posterior midgut invagination reaches the head region along the dorsal side (Fig. 3C) but in the embryos laid by h-s antisense females, sometimes pole cells were still observed in the posterior region (Fig. 3E) and in $20 \%$ of the embryos the gut opening fails to reach the head region, resulting in a hole in the middle of the embryo (Fig. 3F). These embryos failed to develop further and a three-layered germ band was never formed. The lethality of the embryos laid by hs females reached a peak between 18-24 hours after heatshock. At the peak $40-50 \%$ embryos died during the embryo stage. Another $2-3 \%$ embryos laid by hs females could survive to the larval stage, but they had a dark spot inside the body and eventually all died (Table 1 ). The rest of the embryos survived
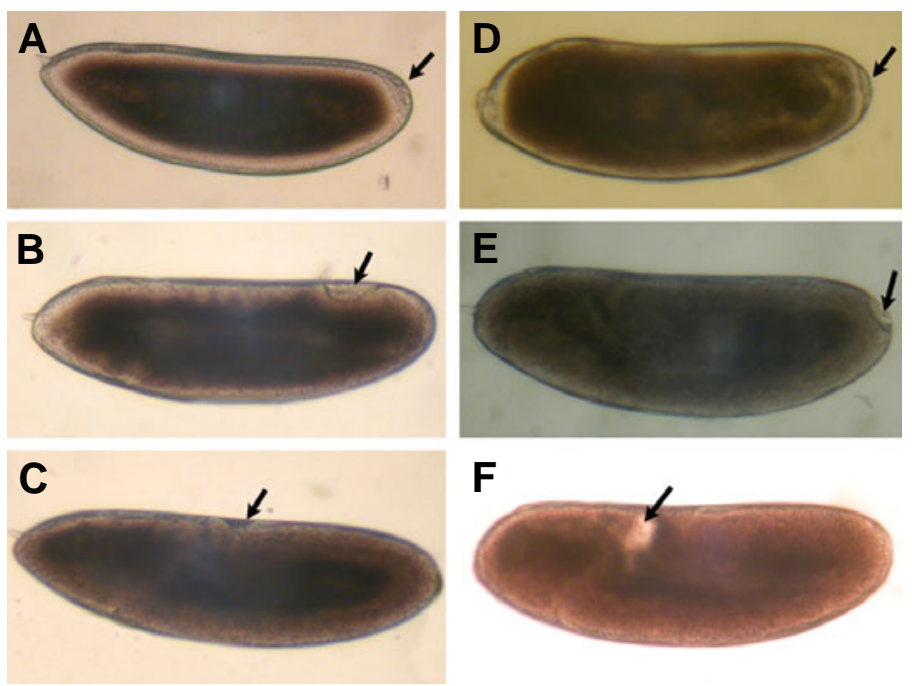

Fig. 3. Abnormal embryogenesis in eggs laid by mothers in which antisense bves is expressed during oogenesis. (A-C) Wild type embryos; (D-F) embryos laid by heatshocked female. (A) Arrow indicates pole cells in the posterior region at stage 5. (B) Arrow indicates posterior midgut invagination at stage 7. (C) Arrow indicates posterior midgut invagination at the anterior at stage 8. (D) Arrow indicates that pole cells remain at the posterior during stage 6-7. (E) Arrow indicates that pole cells are still at the posterior during stage 7-8. (F) Arrow indicates that there is a hole in the middle of the embryo. 
to produce normal adult flies. Survival was lower in line 40 , which affects nurse cell expression to a greater extent, but the phenotypes were similar in both lines.

\section{Discussion}

The popeye genes (Pop1, Pop2 and Pop3) are members of a gene family recently identified in chicken, mouse and human (Andree, et al., 2000; Reese and Bader,1999). We have identified the homologue of one member of this gene family (bves) in Drosophila melanogaster. Its expression during Drosophila oogenesis as well as during embryonic development has been investigated. By reducing the expression levels of bves during oogenesis by expression of its antisense strand we have observed abnormal epithelial movement resulting in the failure of pole cell migration and a high percentage of embryonic death.

bves encodes a putative transmembrane glycoprotein, which is well conserved in evolution. Most similar to Pop1/Bves. Five Pop 1/Bves homologues including bves have been cloned so far from human, mouse, chicken, Drosophila and the mosquito (Andree, et al., 2000; Reese and Bader, 1999; Ensembl, European Bioinformatics Institute,2004). The comparison of Pop1/ Bves protein sequences from 5 species shows that bveshas $59 \%$ identity and $71 \%$ similarity to the mosquito, which is much higher than the identity and similarity compared to vertebrates. Pop1/ Bves proteins all have three putative transmembrane domains and one or two potential glycosylation sites. Both glycosylation of Pop1/Bves protein and its location in the cellular membrane have been shown recently, indicating that they are cell adhesion molecules.

bves is very well conserved in evolution and might therefore have an important function in development. The pop1/Bves knockout mice are viable but have impaired skeletal muscle regeneration after muscle damage by cardiotoxin (Andree, et al., 2002).

bves expression is regulated by the Grk/EGFR signalling pathway. Cellular interaction between the germ line derived oocyte and somatic epithelial follicle cells surrounding the oocyte is required for the development of a mature egg (Schupbach, 1987). Grk/EGFR signalling is involved in the establishment of both the anterior- posterior and dorsal-ventral axes of egg (Gonzalez-Reyes, et al., 1995). During the establishment of the dorsa-ventral axis, grktranscripts together with the oocyte nucleus are located in the anterior-dorsal region of the oocyte and the oocyte sends the Grk signals to adjacent follicle cells. Upon activation of the EGFR downstream signalling pathway, these follicle cells which receive the signals are determined to become dorsal cells. The follicle cells in turn are involved in specifying the dorsal-ventral axis of the subsequent egg. While we were searching for genes downstream of the Grk/EGFR signalling pathway expressed in follicle cells, using a GAL4/UAS enhancer trap system (Deng, et al., 1997), we cloned the bvesgene. A developmental Northern analysis showed that bves is expressed in oogenesis, embryogenesis and postembryogenesis (Fig. 1C). During oogenesis, bves is first activated in nurse cells from stage 5-6 onwards and then in the follicle cells at stage 10. The expression of bves in follicle cells is restricted to those cells which do not receive the Grk signal from oocyte. We found that bveswas expressed in all follicle cells in grkmutant ovaries where there was no Grk signal from the oocyte and that bveswas not expressed in an anterior follicle cells in $f_{s}(1) \mathrm{K} 10$ mutant ovaries where grk transcripts are no longer restricted to anterior dorsal, but are found across the entire anterior of oocyte. Thus, the expression of bves is downstream of the Grk/EGFR signalling pathway.

bves is also expressed in nurse cells and its transcripts are then transported into oocyte. RT-PCR has shown that bves RNA is highly abundant in the $0-2$ hour embryo (Data not shown). Since there is no transcription by this stage, bves RNA must be maternally provided and could be translated and used for early embryogenesis.

Unfortunately there are no bves mutants available neither did we generate any lethal mutants by P-element mobilisation. Thus, in order to investigate the biological function of bves, its antisense strand has been expressed directed by heatshock of pCaS-hsbve-AS lines. Embryos laid by these heatshocked transgenic flies showed a high percentage of embryonic death compared with wild type flies and at the peak, $40-50 \%$ of the embryos died. Another $2-3 \%$ of embryos survived to the larval stage, but eventually died. One of the most obvious mutant phenotypes is the failure of pole cell migration. At stage 7, there was no obvious posterior midgut invagination and with germ band extension, pole cells were left behind at the posterior end of embryos (Fig. 3D,E). Another mutant phenotype was a hole or gap forming between the cephalic furrow and the migrating anterior germ band (Fig. 3F).

The results from the transgenic fly antisense experiment is not consistent with that from the P-element mobilisation. No bves mutants were obtain by $\mathrm{P}$-element mobilisation in our screen. However, it was possible we did not generate any damage to the coding sequence in any line. Searching for bves information in flybase (flybase.bio.indiana.edu), the piggyBac transposon, has been inserted into bvesgene but the flies are still viable and fertile (Bloomington Drosophila Stock Center, 2004). While this result is consistent with our mutagenesis experiment it then makes it less clear why heatshocked bves antisense flies caused the lethality of embryos. One model is that possibly bves is a redundant or non-essential gene. Unfortunately, within species there can be very low levels of sequence similarly between members of the Pop 1/BVESfamily (Osler, et al., 2006) so it is difficult to ascertain if there is a potentially redundant gene in Drosophila based on sequence analysis. If the products of the bves gene can interact with other proteins for cell adhesion, when BVES is knocked out or reduced, perhaps another gene can fulfil the role of bves. Perhaps it is not possible in early embryos to compensate for the lack of maternal product as the product of bvesor its substitute is required prior to transcription of the zygotic genome and the redundant gene product is not maternally stored or not present in sufficient quantities to enable normal embryogenesis to occur. The importance of the maternal products in this effect is also suggested by the higher lethality of embryos in the line where nurse cell expression is reduced to a greater extent than the line affecting largely follicle cell expression. Another possibility is that the piggyBac insertion simply does not knock out or down bves transcription and bves is not redundant but an essential gene for early embryogenesis.

Pop1/Bves protein has been observed in epithelial tissue during organogenesis in other species (Osler and Bader, 2004; Ripley, et al., 2004). Immunostaining of cardiomyocytes cultured in vitroconfirmed the membrane localization of Pop1/Bves in cells 
that participate in cell adhesion (Vasavada, et al., 2004). An in vitro model of corneal wound healing showed that Bves staining was missing at the epithelial surface during cellular migration across the wound, but it reappeared at the points of cell contact during the reinitiation of epithelial continuity (Ripley, et al., 2004). The C-terminal intracellular region of the BVES protein is likely to interact with other proteins but it lacks obvious protein-protein interaction domains (Osler, et al., 2006). The tight junction component ZO-1 interacts with BVES (Osler, et al., 2005). ZO-1 is a scaffold protein that interacts with a number of tight adherens and gap junction proteins (Itoh, et al., 1993; 1999, Fanning, et al., 1998; Barker, et al., 2001) however, just how this protein interacts with BVES is unknown. From the phenotypes observed in our bves antisense RNA experiment, we suggest that bves, as a glycosilated transmembrane protein is required for posterior midgut invagination, adhesion of pole cells to the posterior midgut primordium and contact between the cephalic furrow and the leading edge of the germ band. Hence reduced protein levels, eventually caused the mutant phenotypes we have observed. The need for antibodies to follow the location of bves protein in specific cells during early embryogenesis and changes in the antisense lines is essential.

The function of bves in oogenesis remains unclear. Its expression is restricted to ventral follicle cells by GRK/EGFR signalling. We presume that bves, as an adhesion molecule might more likely be involved in the migration of those ventral follicle cells to cover oocyte than in the establishment of ventral signal essential for embryonic dorsal-ventral pattern. As no mutant phenotype using antisense was found during oogenesis, this hypothesis remains to be investigated.

The key to further progress/investigation of this potentially exciting gene product in cell adhesion and cell migration in embryogenesis in Drosophila will need the generation of good antibodies and mutants.

\section{Materials and Methods}

\section{Fly stocks}

Wild type flies were OrR. Embryo hosts for injection were $w^{1118}$. For other lines refer to papers referenced. grkHK and grkWG (Schüpbach, 1987), fs(1)K10(Wieschaus etal., 1978), UAS-lacZ (Brand and Perrimon, 1993), GAL4 line C648 (Deng et al., 1997). All flies were raised on standard cornmeal-yeast agar medium at $25^{\circ} \mathrm{C}$.

\section{Library screening and DNA sequencing}

$5 \mathrm{~kb}$ of genomic DNA was rescued from GAL4 line C648. The genomic DNA was then used to screen an ovarian cDNA library (Stratagene, Îzap, a gift from Y. Jan, UCSF, USA). One clone was obtained, with an insert of $0.8 \mathrm{~kb}$, which was sequenced. An expressed sequence tag (EST) database search was carried out. Two EST clones (GM07524, LD22978) were found to be similar to the cloned cDNA. These two EST clones were obtained from Berkeley Drosophila Genome Project (BDGP) and sequenced.

\section{Generation of transgenic flies}

A screen mobilising the P-element in the GAL4 line C648 did not generate any lethal mutants in the bves genes, despite generating lethal mutants in other nearby genes. It was therefore necessary to use alternative approaches to investigate the function of this gene. To construct heatshock bves antisense RNA lines, bves cDNA (GM07524) was digested with $E C O R /$ and the fragments were ligated into pCaSpeR- hs vectors in the antisense orientation.. The constructs were then introduced into $w^{1118}$ flies by P-element mediated germline transformation (Spradling, 1986), resulting in the transgenic flies: pCaS-hs-bveAS.

\section{Heatshock treatment of pCaS-hs-bve-AS fly lines}

Flies were cultured at $25^{\circ} \mathrm{C}$ and fed yeast one day before heatshock. Flies were heatshocked for 45 minutes at $39^{\circ} \mathrm{C}$, then put in the $18^{\circ} \mathrm{C}$ incubator for 30 minutes and finally the flies were heatshocked again for 45 minutes at $39^{\circ} \mathrm{C}$. The flies were moved back to $25^{\circ} \mathrm{C}$ and the eggs collected and observed at 4 hours intervals.

\section{Whole mount in situ hybridisation and $\beta$-galactosidase staining}

Whole mount in situ hybridisation and $\beta$-galactosidase staining were as described by Zhao et al. (2000).

\section{Observation of living embryos}

Mutant and wild type embryos were dechorionated by rolling the embryos gently on double-sided sticky tape. Dechorionated embryos were transferred to a slide which had a thin strip of double sided sticky tape on it. The embryos were covered with KELF oil and observed. No defects were observed in wild type embryos.

\section{Acknowledgements}

We thank George Tzolovsky, Kathleen Rothwell, Kevin Leaper and other colleagues in our lab for their help. Shengyin Lin received a faculty studentship from the University of Edinburgh. The research was initially supported by the Wellcome Trust. We are grateful to Hilary Anderson for preparation of the manuscript.

\section{References}

ANDREe, B., FLeige A., ARNOLD H. H. and BRAND T. (2002) Mouse Pop1 is required for muscle regeneration in adult skeletal muscle. $\mathrm{Mol} \mathrm{Ce} / / \mathrm{Biol} .22$ : 1504-12.

ANDREE, B., HILLEMANN, T., KESSLER-ICEKSON, G., SCHMITT-JOHN, T., JOCKUSCH, H., ARNOLD, H.H., BRAND, T. (2000). Isolation and characterization of the novel popeye gene family expressed in skeletal muscle and heart. Dev Biol. 15; 223(2): 371-82.

BELLEN, H.J., O'KANE, C.J., WILSON, C., GROSSNIKLAUS, R.K. and GEHRING, W.J. (1989). P-element-mediated enhancer detection: a versatile method to study development in Drosophila. Genes Dev. 3: 1288-1300.

BRAND, A.H. and PERRIMON, N. (1993). Target gene expression as a means of altering cell fates and generating dominant phenotypes. Development, 118: 401-415.

BARKER, R.J., PRICE, R.L. and GOURDIE, R.G. (2001). Increased co-localization of connexion 43 and ZO-1 in dissociated adult myocytes. Cell Commun Adhes. 8: 205-208

CAMPOS-ORTEGA, J.A. and HARTENSTEIN, V. (1985). The Embryonic development of Drosophila melanogaster. Springer-Verlag, Berlin and Heidelberg.

COSTA, M., SWEETON, D. and WIESCHAUS, E. (1993). Gastrulation in Drosophila: cellular mechanisms of morphogenetic movements. In: M. Bate and A. Martinez-Arias, Editors, The development of Drosophila melanogaster 1, Cold Spring Harbor Laboratory Press, New York, pp. 425-465.

DENG, W.M., ZHAO D., ROTHWELL, K. and BOWNES M.(1997). Analysis of $P[g a \mid 4]$ insertion lines of Drosophila melanogaster as a route to identifying genes important in the follicle cells during oogenesis. Mol Hum Reprod. 3: 85362.

DENG, W.M. and RUOHOLA-BAKER, H. (2000). Laminin A is required for follicle cell oocyte signalling that leads to establishment of the anterior-posterior axis in Drosophila. Curr Bio/10: 683-686.

DIANGELO, J.R., VASAVADA, T.K., CAIN, W. and DUNCAN, M.K. (2001). Production of monoclonal antibodies against chicken Pop1 (BVES). Hybrid Hybridomics. 20: 377-81.

Ensembl, European Bioinformatics Institute, Wellcome Trust Genome Campus, Hinxton, Cambridge (2004). Anopheles Genome Sequencing consortium. 


\section{Lin et al.}

FANNING, A.S., JAMESON, B.J., JESAITIS, L.A. AND ANDERSON, J.M. (1998). The tight junction protein ZO-1 establishes a link between the transmembrane protein occludin and the actin cytoskeleton. J Biol Chem 272: 29745-29753.

GONZALEZ-REYES, A., ELLIOTT, H. and, ST JOHNSTON, D. (1995). Polarization of both major body axes in Drosophilaby gurken-torpedosignalling. Nature375: 654-658.

HITZ, M.P., PANDUR, P., BRAND, T and KUHL, M. (2002) Cardiac specific expression of Xenopus Popeye-1. Mech Dev. 115: 123-6.

ITOH, M., NAGAFUCHI, A., YONEMURA, S., KITANIYASUDA, T. and TSUKITA, S. (1993). The 220-KD protein colocalizing with cadherins in non-epithelial cells is identical to ZO-1, a tight junction-associated protein in epithelial cells: cDNA cloning and immunoelectron microscopy. J Cel/ Biol. 121: 491-502.

ITOH, M., FURUSE, M., MORITA, K., KUBOTA, K., SAITOU, M. AND TSUKITA S. (1999). Direct binding of three tight junction-associated MAGUKs, ZO-1, ZO-2 and ZO-3, with the COOH termini of claudins. J Cell Biol. 147: 1351-1363.

JAGLARZ, M. and HOWARD, K. (1994). Primordial germ cell migration in Drosophila melanogaster is controlled by somatic tissue. Development 120: 83-89.

KNIGHT, R.F., BADER, D.M. and BACKSTROM, J.R. (2003). Membrane topology of Bves/Pop1A, a cell adhesion molecule that displays dynamic changes in cellular distribution during development. J Biol Chem. 29: 32872-9.

KARPEN, G.H. (1994) Position-effect variegation and the new biology of heterochromatin. Curr. Opin. Genet. Dev. 4: 281-291.

MAHOWALD, A.P. (2001). Assembly of the Drosophila germ plasm, Int Rev Cytol. 203:187-213.

OSLER, M.E. and BADER, D.M. (2004). Bves expression during avian embryogenesis. Dev Dyn. 229: 658-67.

OSLER, M.E., CHANG, M.S. AND BADER D.M. (2005). Bves modulates epithelia integrity through an interaction at the tight junction. J Cel/ Sci. 118: 4667-4678.

OSLER, M.E., SMITH, T.K. AND BADER D.M. (2006). Bves, a member of the popeye Domain-containing Gene Family. Dev Dyn. 235: 586-593.

REESE, D.E., ZAVALJEVSKI, M., STREIFF, N.L. and BADER, D. (1999). Bves: A novel gene expressed during coronary blood vessel development. Dev Biol. 209: 159-71

REESE, D.E. and BADER, D.M. (1999). Cloning and expression of hbves, a novel and highly conserved mRNA expressed in the developing and adult heart and skeletal muscle in the human. Mamm. Genome. 10: 913-5.

RIECHMANN, V. and EPHRUSSI, A. (2001). Axis formation during Drosophila oogenesis. Curr. Opin. Genet. Dev. 11: 374-383.
RIPLEY, A.N., CHANG, M.S. and BADER D.M. (2004). Bves is expressed in the epithelial components of the retina, lens and cornea. Invest Ophthalmol Vis Sci. 45: $2475-83$.

ROBERTS, D.B. (1998). Drosophila, A practical approach. second edition (Oxford university press).

SCHUPBACH, T. (1987) Germ line and soma cooperate during oogenesis to establish the dorsoventral pattern of egg shell and embryo in Drosophila melanogaster. Cell. 49: 699-707.

SIMMONS, M.J., RAYMOND, D., GRIMES, C.D., BELINCO, C., HAAKE, B.C., JORDAN, M., LUND, C., OJALA, T.A. and PAPERMSTERET, D. (1996). Repression of Hybrid Dysgenesis in Drosophila melanogaster by heat shock inducible sense and antisense P-element constructs. Genetics. 144: 1529-44.

SPRADLING, A.C. (1986). In Drosophila. A Practical Approach. (ed. D.B.Robert), pp.175-197. Oxford, England: IRL Press.

SPRADLING, A.C. (1993). Developmental genetics of oogenesis. In: M. Bate and A. Martinez-Arias, Editors, The development of Drosophila melanogaster 1, Cold Spring Harbor Laboratory Press, New York pp. 1-70.

TELFER, W. (1975). Development and physiology of the oocyte-nurse cell syncitium. Adv. Insect Physiol. 11: 223-319.

VASAVADA, T.K., DIANGELO, J.R. and DUNCAN, M.K. (2004). Developmental expression of Pop1/Bves. J Histochem Cytochem. 52: 371-78.

WADA, A.M., REESE, D.E. and BADER, D.M. (2001). Bves: prototype of a new class of cell adhesion molecules expressed during coronary artery development. Development. 128: 2085-93.

WARRIOR, R. (1994). Primordial germ cell migration and the assembly of the Drosophila embryonic gonad. Dev Biol., 166: 180-94.

WIESCHAUS, E., MARSH, J and GEHRING, W. (1978). fs(1) K10, a germline dependent female sterile mutation causing abnormal chorion morphology in Drosophila melanogaster. Roux's Arch.Dev.Biol. 184: 75-82.

ZHAO, D., CLYDE, D., BOWNES, M. (2000). Expression of fringeis down regulated by Gurken/Epidermal Growth Factor Receptor signalling and is required for the morphogenesis of ovarian follicle cells. J. Cell Science 113: 3781-3794.

Received: 5 th October 2005 Reviewed by Referees: 24th April 2006 Modified by Authors and Accepted for Publication: 24th October 2006 Published Online: 24th November 2006 